\title{
Addition of C-nucleophiles to 5-phenylpyrimidin-2(1H)-ones and 6-phenyl-1,2,4-triazin-3(2H)-one
}

\author{
Ilya N. Egorov, ${ }^{\text {a }}$ Tatyana A. Tseitler, ${ }^{a}$ Grigory V. Zyryanov,,${ }^{\text {a,b }}$ Vladimir L. Rusinov, ${ }^{\text {a }}$ and \\ Oleg N. Chupakhin ${ }^{\mathrm{a}, \mathrm{b}}$ \\ ${ }^{a}$ Ural Federal University, Department of Organic Chemistry, \\ Mira St. 19, Ekaterinburg, 620 002, Russia \\ ${ }^{b}$ Institute of Organic Synthesis Ural Branch of Russian Academy of Sciences, \\ S. Kovalevskoy St. 22, Ekaterinburg, 620 041, Russia \\ E-mail: i.n.egorov@gmail.com, chupakhin@ios.uran.ru
}

\begin{abstract}
Addition reactions of $\mathrm{C}$-nucleophiles to the $\mathrm{C}=\mathrm{N}$ bond of 5-phenyl- and 1-methyl-5phenylpyrimidin-2(1H)-ones $\mathbf{1 a}, \mathbf{b}$ and 6-phenyl-1,2,4-triazin-3(2H)-one $\mathbf{2}$ were investigated. 1a,b and 2 furnished addition products with indoles; 1a also added $N$-methylpyrrole. Only 2 added thiophene and methyl ketones, and reacted with alkyl halides and acetone forming products resulting both from alkylation and from addition of acetone at positions 2 and 5 , respectively.
\end{abstract}

Keywords: Pyrimidinones, 1,2,4-triazinones, indoles, nucleophilic addition reactions, azaFriedel-Crafts reactions

\section{Introduction}

Pyrimidinones and 1,2,4-triazinones are analogs of the pyrimidine bases of nucleic acids. Therefore, they possess a big potential in the search for new biologically active compounds. They are interesting as potential agonists and antagonists of pyrimidine derivatives, widespread in nature. ${ }^{1}$ Thus, 5-substituted pyrimidin-2-ones exhibit metaphase-arresting activity, ${ }^{2}$ their nucleoside derivatives show anticancer ${ }^{3}$ and antivirus activity. ${ }^{4}$ Derivatives of 1,2,4-triazin$3(2 H)$-ones are also of interest as anticancer ${ }^{5}$, antivirus ${ }^{6}$ and antibacterial drugs. ${ }^{7}$

Pyrimidin-2-ones and 1,2,4-triazin-3(2H)-ones are $\pi$-deficient heterocycles susceptible to addition reactions of C-nucleophiles. ${ }^{8}$ Pyrimidin-2-ones add organometallic compounds ${ }^{9}$ and indoles. ${ }^{10}$ Also known are reactions of 6-phenyl-1,2,4-triazin-3(2H)-one 2 with N-nucleophiles ${ }^{11}$ and some $\mathrm{C}$ - and O-nucleophiles. ${ }^{12}$ 
In this work we report on different ways for introducing C-nucleophiles into 5phenylpyrimidin-2(1H)-ones 1a,b and its 6-aza analog, 6-phenyl-1,2,4-triazin-3(2H)-one 2 via direct $\mathrm{C}-\mathrm{C}$ coupling (without using organometallic compounds that would require low temperatures and an inert atmosphere). Previously, it has been reported that unsubstituted, protonated pyrimidines in benzene/trifluoroacetic acid solution react with $\mathrm{C}$-nucleophiles to give addition products. ${ }^{13}$ It has been shown also that 6-phenyl-1,2,4-triazin-3(2H)-one 2 reacts with various C-nucleophiles without activation, simply upon heating. ${ }^{12 \mathrm{~b}}$ These reactions constitute socalled aza-Friedel-Crafts reactions of nucleophilic hetarenes, but can be considered also as nucleophilic additions onto conjugated $\mathrm{C}=\mathrm{N}$ double bonds in the presence of Lewis ${ }^{14}$ and Bronsted $^{15}$ acids. Pyrimidin-2(1H)-ones and 1,2,4-triazin-3(2H)-ones contain such electrophilic functionalities capable of participating in aza-Friedel-Crafts reactions under similar conditions.

\section{Results and Discussion}

First, we studied the interactions of 5-phenylpyrimidin-2(1H)-ones 1a,b and 6-phenyl-1,2,4triazin-3(2H)-one 2 with indole, a common nucleophile for aza-Friedel-Crafts reactions. (Scheme 1).<smiles>[R]n1[X]c(-c2ccccc2)cnc1=O</smiles>

1a,b, 2

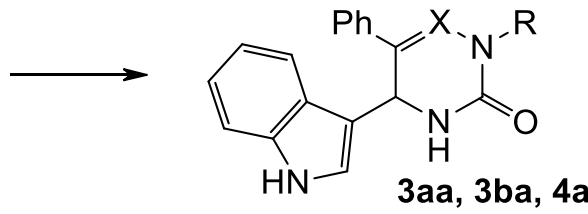

\begin{tabular}{l|ll} 
& $\mathrm{X}$ & $\mathrm{R}$ \\
\hline 1a, 3aa & $\mathrm{CH}$ & $\mathrm{H}$ \\
1b, 3ba & $\mathrm{CH}$ & $\mathrm{Me}$ \\
2, 4a & $\mathrm{N}$ & $\mathrm{H}$
\end{tabular}

\section{Scheme 1}

The reaction of $\mathbf{1 a}$ with indole in refluxing DMF as well as in a mixture of dichloromethane, ethanol and trifluoroacetic acid (or triflic acid) at room temperature gave no product. The reaction carried out in refluxing acetic acid or in chloroform/trifluoroacetic acid at room temperature afforded 3aa in moderate yields (42\% and 28\%, respectively). The reaction of 2 with indole in refluxing DMF or in refluxing acetic acid gave $\mathbf{4 a}$ in high yields $\mathbf{7 5 \%}$ and $86 \%$, respectively). Carrying out the reaction in chloroform/trifluoroacetic acid at room temperature afforded 4a with lower yield (48\%). Attempts to add indole to azinones 1a,b, and 2 under conditions typical for Friedel-Crafts reactions, namely activation by Lewis acids such as $\mathrm{BF}_{3} \cdot \mathrm{OMe}_{2}, \mathrm{Cu}(\mathrm{OTf})_{2}$, or $\mathrm{Co}(\mathrm{OTf})_{2}$ in methanol, acetonitrile or acetic acid were unsuccessful in most cases. The reaction did not occur at room temperature, heating gave rise to tarring. The use of a Lewis acid was successful only in the reaction of 2 with indole in acetic acid and in the presence of $\mathrm{Co}(\mathrm{OTf})_{2}$ yielding 4a (15\%). In most cases, 6-phenyl-1,2,4-triazin-3(2H)-one 2 appeared to be more reactive than 5-phenylpyrimidin-2(1H)-one 1a. 
From the reactions of 5-phenylpyrimidin-2(1H)-ones 1a,b and 6-phenyl-1,2,4-triazin3(2H)-one $\mathbf{2}$ with indoles, addition products $\mathbf{3 a a}-\mathbf{a c}, \mathbf{3 b a}-\mathbf{b c}, \mathbf{4 a - c}$ were isolated (Table 1). 1a reacted with $N$-methylpyrrole affording $\mathbf{3 a d}$, but there was no addition product isolated from the reaction of azinones $\mathbf{1 b}, \mathbf{2}$ with pyrroles because the latter tend to polymerize in acidic solutions.

Table 1. Reaction of C-nucleophiles with 1a,b and 2; formation of addition products $\mathbf{3}$ and $\mathbf{4}$
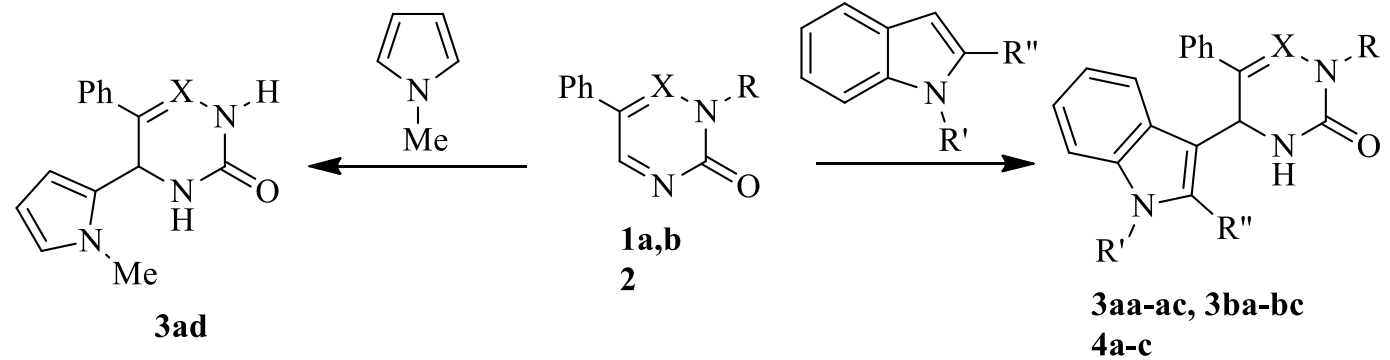

\begin{tabular}{cccccccc}
\hline Nu & $\begin{array}{c}\text { Starting } \\
\text { material }\end{array}$ & $\mathrm{X}$ & $\mathrm{R}$ & $\mathrm{R}{ }^{\prime}$ & $\mathrm{R} ”$ & Product & Yield [\%] \\
\hline 1H-indol-3-yl & $\mathbf{1 a}$ & $\mathrm{CH}$ & $\mathrm{H}$ & $\mathrm{H}$ & $\mathrm{H}$ & $\mathbf{3 a a}$ & 28 \\
1H-indol-3-yl & $\mathbf{2}$ & $\mathrm{N}$ & $\mathrm{H}$ & $\mathrm{H}$ & $\mathrm{H}$ & $\mathbf{4 a}$ & 48 \\
$1 H$-indol-3-yl & $\mathbf{1 b}$ & $\mathrm{CH}$ & $\mathrm{Me}$ & $\mathrm{H}$ & $\mathrm{H}$ & $\mathbf{3 b a}$ & 84 \\
1-methyl1 $H$-indol-3-yl & $\mathbf{1 a}$ & $\mathrm{CH}$ & $\mathrm{H}$ & $\mathrm{Me}$ & $\mathrm{H}$ & $\mathbf{3 a b}$ & 42 \\
1-methyl1 $H$-indol-3-yl & $\mathbf{2}$ & $\mathrm{N}$ & $\mathrm{H}$ & $\mathrm{Me}$ & $\mathrm{H}$ & $\mathbf{4 b}$ & 63 \\
1-methyl1 $H$-indol-3-yl & $\mathbf{1 b}$ & $\mathrm{CH}$ & $\mathrm{Me}$ & $\mathrm{Me}$ & $\mathrm{H}$ & $\mathbf{3 b b}$ & 73 \\
2-methyl1 $H$-indol-3-yl & $\mathbf{1 a}$ & $\mathrm{CH}$ & $\mathrm{H}$ & $\mathrm{H}$ & $\mathrm{Me}$ & $\mathbf{3 a c}$ & 55 \\
2-methyl1 $H$-indol-3-yl & $\mathbf{2}$ & $\mathrm{N}$ & $\mathrm{H}$ & $\mathrm{H}$ & $\mathrm{Me}$ & $\mathbf{4 c}$ & 48 \\
2-methyl1 $H$-indol-3-yl & $\mathbf{1 b}$ & $\mathrm{CH}$ & $\mathrm{Me}$ & $\mathrm{H}$ & $\mathrm{Me}$ & $\mathbf{3 b c}$ & 50 \\
1-methyl-1 $H$-pyrrol-2-yl & $\mathbf{1 a}$ & $\mathrm{CH}$ & $\mathrm{H}$ & - & - & $\mathbf{3 a d}$ & 28 \\
\hline
\end{tabular}

We also investigated the reactions of $\mathbf{1 a}, \mathbf{b}$ and $\mathbf{2}$ with thiophene and methyl ketones. In refluxing acetic acid or in chloroform in the presence of trifluoroacetic acid no addition products were formed, presumably due to the lower nucleophilicity of these compounds as compared to indoles. However, in refluxing acetic acid and catalyzed by $\mathrm{BF}_{3} \cdot \mathrm{OMe}_{2}$, 6-phenyl-1,2,4-triazin$3(2 H)$-one $\mathbf{2}$ afforded addition products $\mathbf{4 d}-\mathbf{4 g}$ (Table 2).

Unlike the addition of thiophene to $\mathbf{2}$ yielding $\mathbf{4 d}$, 2 -acetothienone adds to $\mathbf{2}$ with the methyl group producing 4f; obviously, the acetyl group of 2-acetothienone lowers the nucleophilicity of the thiophene ring and the enol tautomer of the acetyl group is acting as nucleophile. Similarly, the methyl groups of acetophenone and acetone add to the $\mathrm{C}=\mathrm{N}$ functionality in $\mathbf{2}$ affording products $\mathbf{4 e}$ and $\mathbf{4 g}$, respectively. 
Table 2. Conversion of $\mathbf{2}$ into addition products $\mathbf{4 d}-\mathbf{g}$

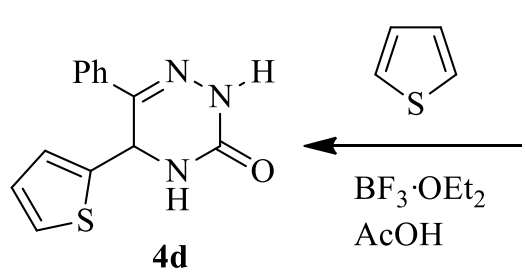<smiles>O=c1ncc(-c2ccccc2)n[nH]1</smiles><smiles></smiles><smiles>[R]C(=O)CC1NC(=O)NN=C1Pc1ccccc1</smiles>

\begin{tabular}{llcc}
\hline $\mathrm{Nu}$ & $\mathrm{R}$ & Product & Yield [\%] \\
\hline thiophen-2-yl & - & $\mathbf{4 d}$ & 43 \\
2-oxo-2-phenyethyl & $\mathrm{Ph}$ & $\mathbf{4 e}$ & 59 \\
2-oxo-2-(thiophen-2-yl)ethyl & thiophen-2-yl & $\mathbf{4 f}$ & 41 \\
2-oxopropyl & $\mathrm{Me}$ & $\mathbf{4 g}$ & 42 \\
\hline
\end{tabular}

5-Phenylpyrimidin-2(1H)-ones $\mathbf{1 a , b}$ did not undergo addition reactions with the reactants listed in Table 2 under the conditions applied to their reactions with 2 ; also the reaction in refluxing acetone in the presence of hydrochloric acid failed. ${ }^{16}$

The ${ }^{1} \mathrm{H}$ and ${ }^{13} \mathrm{C}$ NMR data of $\mathbf{4 e}$ did not allow an unambiguous structure proof; therefore, the structure of $\mathbf{4 e}$ was confirmed by X-ray analysis (Figure 1).

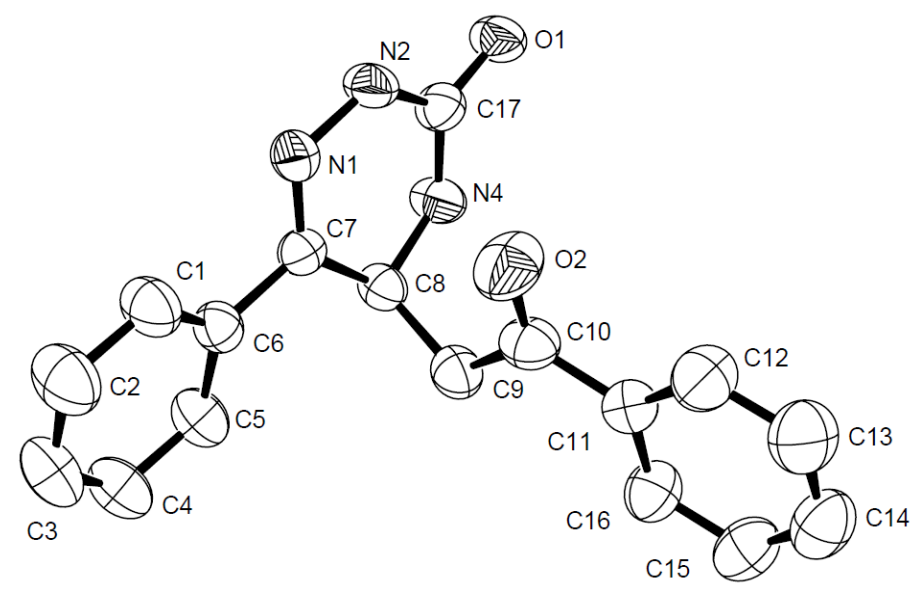

Figure 1. ORTEP diagram of $4 e^{17 \mathrm{a}}$

Treatment of 6-phenyl-1,2,4-triazin-3(2H)-one 2 with alkyl halides in aqueous acetone and in the presence of sodium hydroxide formed 2-alkyl-5-(2-oxopropyl)-6-phenyl-4,5-dihydro1,2,4-triazin-3(2H)-ones 5a-d resulting both from alkylation and from addition of acetone at positions 2 and 5, respectively (Table 3, Figure 2). Under the same conditions, 5phenylpyrimidin-2(1H)-one 1a did not react with acetone. 
Table 3. Reaction of $\mathbf{2}$ with alkyl halides and acetone forming alkylated addition products $\mathbf{5 a - d}$

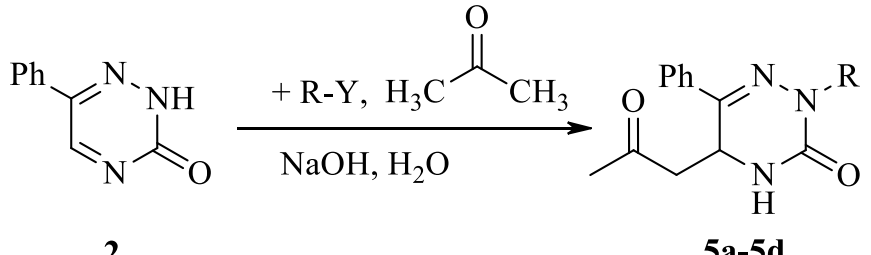

5a-5d

\begin{tabular}{llccc}
\hline $\mathrm{R}$ & $\mathrm{Y}$ & $\mathrm{R}-\mathrm{Y}$ [equiv.] & Product & Yield [\%] \\
\hline $\mathrm{CH}_{3}$ & $\mathrm{I}$ & 10 & $\mathbf{5 a}$ & 34 \\
$\mathrm{CH}_{3} \mathrm{CH}_{2}$ & $\mathrm{I}$ & 2 & $\mathbf{5 b}$ & 38 \\
$\mathrm{CH}_{3} \mathrm{CH}_{2} \mathrm{CH}_{2}$ & $\mathrm{Br}$ & 1.2 & $\mathbf{5 c}$ & 12 \\
$\mathrm{PhCH}_{2}$ & $\mathrm{Cl}$ & 1.2 & $\mathbf{5 d}$ & 14 \\
\hline
\end{tabular}

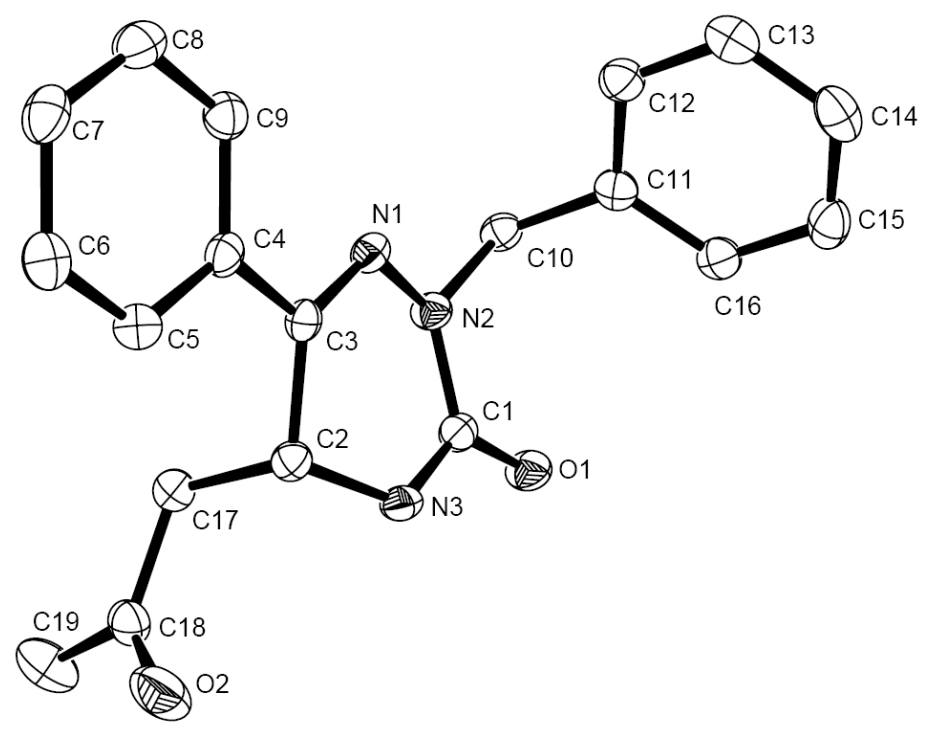

Figure 2. ORTEP diagram of $\mathbf{5 d} .^{17 b}$

It should be noted that 6-phenyl-1,2,4-triazin-3(2H)-one 2 adds N-nucleophiles ${ }^{11}$ and Onucleophiles. ${ }^{12 \mathrm{a}}$ By contrast, 5-phenylpyrimidin-2(1H)-one $\mathbf{1 a}$ did not react with cyclic amines (morpholine, piperidine) neither upon heating with pure amines nor in the presence of sulfur as oxidant; 1a is also unreactive toward aliphatic alcohols both under basic and acidic conditions (in the presence of triethylamine and trifluoroacetic acid, respectively). 


\section{Experimental Section}

General. $\mathbf{1} \mathbf{b}^{18}$ and $\mathbf{2} \mathbf{a}^{19}$ were synthesized by known methods; other reactants are commercially available. TLC analysis was performed on Merck silica gel $60 \mathrm{~F}_{254}$ plates and visualized by exposure to UV light. ${ }^{1} \mathrm{H}$ and ${ }^{13} \mathrm{C}$ NMR spectra were recorded on a Bruker DRX-400 spectrometer, using tetramethylsilane (TMS) as an internal standard. X-Ray analysis including data collection, cell refinement and data reduction was carried out with an Oxford Diffraction Xcalibur S CCD diffractometer using CrysAlisPro software package. ${ }^{20}$ The structures were resolved using SHELXS-97 and refined by full-matrix least-squares procedure on $\mathrm{F}^{2}$ with SHELXL-97. ${ }^{21}$

5-Phenyl-2(1H)-pyrimidinone (1a). A mixture of phenylmalondialdehyde hydrate (1.50 g, 9 $\mathrm{mmol})$, urea (1.084 g, $18 \mathrm{mmol})$ and $p$-toluenesulfonic acid (100 $\mathrm{mg}$ ) was dissolved in toluene $(50 \mathrm{~mL})$ and refluxed using a Dean-Stark trap for $40 \mathrm{~min}$. Then, toluene was decanted and the insoluble gummy residue was crystallized from ethanol to give a white powder 1a (0.62 $\mathrm{g}, 40 \%)$; mp $239{ }^{\circ} \mathrm{C}$ (lit. ${ }^{22} \mathrm{mp} 237{ }^{\circ} \mathrm{C}$ ). ${ }^{1} \mathrm{H}$ NMR (400 MHz, DMSO- $d_{6} \square: \delta 7.28-7.31$ (m, 1H, Ph), 7.38$7.42(\mathrm{~m}, 2 \mathrm{H}, \mathrm{Ph}), 7.53-7.55$ (m, 2H, Ph), 8.51 (s, 2H), 12.14 (s, 1H, NH). ${ }^{1} \mathrm{H}$ NMR spectral data correspond to those given in the literature. ${ }^{23}$

\section{General procedure for the synthesis of (3) and (4a-c)}

To a suspension of $\mathbf{1 a}, \mathbf{b}$ or $\mathbf{2}(150 \mathrm{mg})$ in chloroform $(5 \mathrm{~mL})$ was added trifluoroacetic acid (100 $\mu \mathrm{L})$ and the nucleophilic reactant (1.1 equiv.). The mixture was stirred at ambient temperature for $18 \mathrm{~h}$. Then, triethylamine $(100 \mu \mathrm{L})$ was added, the mixture was evaporated, and the residue was crystallized from ethanol.

4-(1H-Indol-3-yl)-5-phenyl-3,4-dihydropyrimidin-2(1H)-one (3aa). 1a (150 mg, $0.871 \mathrm{mmol})$ and indole (112 mg, $0.956 \mathrm{mmol})$ gave grey crystals 3aa $(71 \mathrm{mg}, 28 \%)$. Alternatively, the reaction was carried out in acetic acid $(5 \mathrm{~mL})$ and refluxed for $1-3 \mathrm{~h}$ affording 3aa (106 $\mathrm{mg}$, 42\%); mp 248-249 ${ }^{\circ} \mathrm{C} . \mathrm{R}_{\mathrm{f}}=0.45$ (EtOAc). ${ }^{1} \mathrm{H}$ NMR (400 MHz, DMSO-d $): \delta 55.63$ (d, $J=2.8$ $\mathrm{Hz}, 1 \mathrm{H}), 6.74(\mathrm{~d}, J=5.3 \mathrm{~Hz}, 1 \mathrm{H}), 6.95-7.04(\mathrm{~m}, 3 \mathrm{H}), 7.06-7.14(\mathrm{~m}, 4 \mathrm{H}), 7.24-7.26(\mathrm{~m}, 2 \mathrm{H})$, $7.30(\mathrm{~d}, J=8.0 \mathrm{~Hz}, 1 \mathrm{H}), 7.75(\mathrm{~d}, J=7.8 \mathrm{~Hz}, 1 \mathrm{H}), 8.52(\mathrm{~d}, J=3.6 \mathrm{~Hz}, 1 \mathrm{H}, \mathrm{NH}), 10.75(\mathrm{~s}, 1 \mathrm{H}$, $\mathrm{NH}) .{ }^{13} \mathrm{C}$ NMR $\left(100 \mathrm{MHz}, \mathrm{DMSO}-d_{6}\right): \delta 48.9,110.5,111.5,117.1,118.6,119.2,121.0,122.5$, 123.6, 124.0, 125.1, 125.3, 128.2, 136.6, 136.7, 152.8. Anal. found: C, 74.75; H, 5.34; N, 14.43. Calcd. for $\mathrm{C}_{18} \mathrm{H}_{15} \mathrm{~N}_{3} \mathrm{O}: \mathrm{C}, 74.72 ; \mathrm{H}, 5.23 ; \mathrm{N}, 14.52$.

4-(1-Methyl-1H-indol-3-yl)-5-phenyl-3,4-dihydropyrimidin-2(1H)-one (3ab). 1a (150 mg, $0.871 \mathrm{mmol}$ ) and 1-methylindole $(126 \mathrm{mg}, 0.961 \mathrm{mmol})$ gave yellow crystals 3ab (111 $\mathrm{mg}$, 42\%); mp 289-290 ${ }^{\circ} \mathrm{C} . \mathrm{R}_{\mathrm{f}}=0.4$ (EtOAc). ${ }^{1} \mathrm{H}$ NMR (400 MHz, DMSO-d $): \delta 3.71$ (s, 3H, $\left.\mathrm{NCH}_{3}\right), 5.61(\mathrm{~d}, J=2.8 \mathrm{~Hz}, 1 \mathrm{H}), 6.75(\mathrm{~d}, J=5.4 \mathrm{~Hz}, 1 \mathrm{H}), 6.99-7.06(\mathrm{~m}, 3 \mathrm{H}), 7.08-7.18(\mathrm{~m}, 4 \mathrm{H})$, 7.24-7.30 (m, 3H), 7.78 (d, $J=7.9 \mathrm{~Hz}, 1 \mathrm{H}), 8.53$ (d, $J=3.8 \mathrm{~Hz}, 1 \mathrm{H}, \mathrm{NH}) .{ }^{13} \mathrm{C} \mathrm{NMR}(100 \mathrm{MHz}$, DMSO- $\left.d_{6}\right): \delta 32.2,48.5,109.7,110.5,116.3,118.7,119.4,121.2,122.7,123.9,125.4,125.5$, 
127.7, 128.2, 136.6, 137.0, 152.8. Anal. found: $\mathrm{C}, 75.20 ; \mathrm{H}, 5.73 ; \mathrm{N}, 13.66$. Calcd. for $\mathrm{C}_{19} \mathrm{H}_{17} \mathrm{~N}_{3} \mathrm{O}: \mathrm{C}, 75.23 ; \mathrm{H}, 5.65 ; \mathrm{N}, 13.85$.

4-(2-Methyl-1H-indol-3-yl)-5-phenyl-3,4-dihydropyrimidin-2(1H)-one (3ac). $1 \mathrm{a}$ (150 mg, $0.871 \mathrm{mmol}$ ) and 2-methylindole (126 mg, $0.961 \mathrm{mmol})$ gave grey crystals 3ac (145 mg, 55\%); mp 239-240 ${ }^{\circ} \mathrm{C} .{ }^{1} \mathrm{H}$ NMR (400 MHz, DMSO-d6): $\delta 2.42\left(\mathrm{~s}, 3 \mathrm{H}, \mathrm{CH}_{3}\right), 5.73(\mathrm{~d}, J=1.8 \mathrm{~Hz}, 1 \mathrm{H})$, $6.58(\mathrm{~d}, J=5.3 \mathrm{~Hz}, 1 \mathrm{H}), 6.80(\mathrm{~s}, 1 \mathrm{H}), 6.84-6.93(\mathrm{~m}, 2 \mathrm{H}), 6.97(\mathrm{t}, J=7.2 \mathrm{~Hz}, 1 \mathrm{H}), 7.08(\mathrm{t}, J=7.7$ $\mathrm{Hz}, 2 \mathrm{H}), 7.14-7.19(\mathrm{~m}, 3 \mathrm{H}), 7.56(\mathrm{~d}, J=7.8 \mathrm{~Hz}, 1 \mathrm{H}), 8.51(\mathrm{~d}, J=3.9 \mathrm{~Hz}, 1 \mathrm{H}, \mathrm{NH}), 10.61$ (s, $1 \mathrm{H}$, $\mathrm{NH}) .{ }^{13} \mathrm{C}$ NMR $\left(100 \mathrm{MHz}, \mathrm{DMSO}-d_{6}\right): \delta 11.9,49.1,110.5,110.9,113.7,118.9,119.0,120.5$, 122.9, 124.6, 125.9, 126.9, 128.6, 133.0, 135.7, 137.7, 152.9. Anal. found: C, 75.05; H, 5.73; N, 13.96. Calcd. for $\mathrm{C}_{19} \mathrm{H}_{17} \mathrm{~N}_{3} \mathrm{O}: \mathrm{C}, 75.23 ; \mathrm{H}, 5.65 ; \mathrm{N}, 13.85$.

4-(1-Methyl-1H-pyrrol-2-yl)-5-phenyl-3,4-dihydropyrimidin-2(1H)-one (3ad). 1a (150 mg, $0.871 \mathrm{mmol}$ ) and $N$-pyrrole (71 mg, $0.958 \mathrm{mmol}$ ) gave grey crystals 3ad (62 $\mathrm{mg}, 28 \%$ ); $\mathrm{mp} 205-$ $206{ }^{\circ} \mathrm{C} .{ }^{1} \mathrm{H}$ NMR (400 MHz, DMSO-d $)$ ): $\delta 3.55$ (s, 3H, NCH$), 5.14(\mathrm{~d}, J=2.9 \mathrm{~Hz}, 1 \mathrm{H}), 5.95(\mathrm{t}$, $J=2.2 \mathrm{~Hz}, 1 \mathrm{H}), 6.47(\mathrm{~d}, J=2.2 \mathrm{~Hz}, 2 \mathrm{H}), 6.65(\mathrm{~d}, J=5.3 \mathrm{~Hz}, 1 \mathrm{H}), 6.94(\mathrm{~s}, 1 \mathrm{H}), 7.04(\mathrm{t}, J=7.3$ $\mathrm{Hz}, 1 \mathrm{H}), 7.17(\mathrm{t}, J=7.8 \mathrm{~Hz}, 2 \mathrm{H}), 7.26(\mathrm{~d}, J=8.1 \mathrm{~Hz}, 2 \mathrm{H}), 8.34(\mathrm{~d}, J=3.6 \mathrm{~Hz}, 1 \mathrm{H}, \mathrm{NH}) .{ }^{13} \mathrm{C}$ NMR (100 MHz, DMSO-d6): $\delta$ 35.5, 49.1, 106.5, 111.8, 118.9, 121.7, 122.2, 123.7, 125.3, 126.3, 128.3, 136.7, 153.3. Anal. found: $\mathrm{C}, 71.32 ; \mathrm{H}, 5.88 ; \mathrm{N}, 16.74$. Calcd. for $\mathrm{C}_{15} \mathrm{H}_{15} \mathrm{~N}_{3} \mathrm{O}$ : $\mathrm{C}$, $71.13 ; \mathrm{H}, 5.97 ; \mathrm{N}, 16.59$.

1-Methyl-4-(1H-indol-3-yl)-5-phenyl-3,4-dihydropyrimidin-2(1H)-one (3ba). $1 \mathrm{~b}$ (150 mg, $0.806 \mathrm{mmol})$ and indole $(104 \mathrm{mg}, 0.888 \mathrm{mmol})$ gave colorless crystals $3 \mathrm{ba}(222 \mathrm{mg}$, 84\%). The reaction was also carried out in a mixture of methylene chloride $(5 \mathrm{~mL})$, ethanol $(2 \mathrm{~mL})$, and trifluoromethanesulfonic acid $(100 \mu \mathrm{L})$ with stirring for $18 \mathrm{~h}$ to furnish $3 \mathbf{b a}(217 \mathrm{mg}, 82 \%)$; mp $174{ }^{\circ} \mathrm{C} . \mathrm{R}_{\mathrm{f}}=0.4$ (EtOAc). ${ }^{1} \mathrm{H}$ NMR $\left(400 \mathrm{MHz}, \mathrm{DMSO}-d_{6} \square\right): \delta 2.78\left(\mathrm{~s}, 3 \mathrm{H}, \mathrm{NCH}_{3}\right), 5.63(\mathrm{~s}, 1 \mathrm{H})$, $6.69(\mathrm{~d}, J=5.3 \mathrm{~Hz}, 1 \mathrm{H}), 6.95-7.05(\mathrm{~m}, 3 \mathrm{H}), 7.12(\mathrm{t}, J=7.7 \mathrm{~Hz}, 2 \mathrm{H}), 7.25-7.31(\mathrm{~m}, 4 \mathrm{H}), 7.67$ (d, $J=7.9 \mathrm{~Hz}, 1 \mathrm{H}), 8.74(\mathrm{~d}, J=5.3 \mathrm{~Hz}, 1 \mathrm{H}, \mathrm{NH}), 10.89(\mathrm{~s}, 1 \mathrm{H}, \mathrm{NH}) .{ }^{13} \mathrm{C}$ NMR $(100 \mathrm{MHz}, \mathrm{DMSO}-$ $\left.d_{6}\right): \delta 32.1,55.8,110.6,111.7,113.7,118.88,118.90,121.0,121.6,123.9,125.0,125.3,125.4$, 128.1, 136.4, 136.5, 152.4. Anal. found: $\mathrm{C}, 75.02 ; \mathrm{H}, 5.57 ; \mathrm{N}, 13.91$. Calcd. for $\mathrm{C}_{19} \mathrm{H}_{17} \mathrm{~N}_{3} \mathrm{O}$ : $\mathrm{C}$, 75.23; H, 5.65; N, 13.85 .

1-Methyl-4-(1-methyl-1H-indol-3-yl)-5-phenyl-3,4-dihydropyrimidin-2(1H)-one (3bb). $1 b$ (150 mg, $0.806 \mathrm{mmol})$ and 1-methylindole $(116 \mathrm{mg}, 0.886 \mathrm{mmol})$ gave colorless crystals $3 \mathbf{b b}$ (202 mg, 73\%); mp 207-208 ${ }^{\circ} \mathrm{C} . \mathrm{R}_{f}=0.45$ (EtOAc). ${ }^{1} \mathrm{H}$ NMR (400 MHz, DMSO- $d_{6} \square: \delta 2.78$ (s, $\left.3 \mathrm{H}, \mathrm{NCH}_{3}\right), 3.73\left(\mathrm{~s}, 3 \mathrm{H}, \mathrm{NCH}_{3}\right), 5.62(\mathrm{~s}, 1 \mathrm{H}), 6.70(\mathrm{~d}, J=5.4 \mathrm{~Hz}, 1 \mathrm{H}), 6.97-7.03(\mathrm{~m}, 2 \mathrm{H}), 7.09-$ $7.13(\mathrm{~m}, 3 \mathrm{H}), 7.24-7.30(\mathrm{~m}, 4 \mathrm{H}), 7.70(\mathrm{~d}, J=8.0 \mathrm{~Hz}, 1 \mathrm{H}), 8.75(\mathrm{~d}, J=5.3 \mathrm{~Hz}, 1 \mathrm{H}, \mathrm{NH}) .{ }^{13} \mathrm{C}$ NMR (100 MHz, DMSO-d6): $\delta$ 32.2, 32.3, 55.3, 109.9, 110.7, 113.0, 119.0, 119.1, 121.2, 121.7 , 123.9, 125.4, 125.8, 128.2, 129.0, 136.4, 136.8, 152.4. Anal. found: C, 75.58; H, 6.28; N, 12.96. Calcd. for $\mathrm{C}_{20} \mathrm{H}_{19} \mathrm{~N}_{3} \mathrm{O}: \mathrm{C}, 75.69 ; \mathrm{H}, 6.03 ; \mathrm{N}, 13.24$.

1-Methyl-4-(2-methyl-1H-indol-3-yl)-5-phenyl-3,4-dihydropyrimidin-2(1H)-one (3bc). $1 \mathrm{~b}$ $(150 \mathrm{mg}, 0.806 \mathrm{mmol})$ and 2-methylindole $(116 \mathrm{mg}, 0.886 \mathrm{mmol})$ gave grey crystals $3 \mathrm{bc}(138$ $\mathrm{mg}, 50 \%)$; mp $276{ }^{\circ} \mathrm{C} .{ }^{1} \mathrm{H}$ NMR (400 MHz, DMSO-d $\left.d_{6}\right): \delta 2.44\left(\mathrm{~s}, 3 \mathrm{H}, \mathrm{CH}_{3}\right), 2.67\left(\mathrm{~s}, 3 \mathrm{H}, \mathrm{NCH}_{3}\right)$, $5.70(\mathrm{~s}, 1 \mathrm{H}), 6.57(\mathrm{~d}, J=5.3 \mathrm{~Hz}, 1 \mathrm{H}), 6.87-6.95(\mathrm{~m}, 2 \mathrm{H}), 6.96-6.70(\mathrm{~m}, 1 \mathrm{H}), 7.08-7.12(\mathrm{~m}, 2 \mathrm{H})$, 
7.16-7.20 (m, 3H), $7.54(\mathrm{~d}, J=7.6 \mathrm{~Hz}, 1 \mathrm{H}), 8.72(\mathrm{~d}, J=5.3 \mathrm{~Hz}, 1 \mathrm{H}, \mathrm{NH}), 10.71(\mathrm{~s}, 1 \mathrm{H}, \mathrm{NH})$. ${ }^{13} \mathrm{C}$ NMR (100 MHz, DMSO-d $)$ : $\delta$ 11.3, 31.4, 55.1, 109.5, 109.9, 110.6, 118.2, 118.6, 120.1, 121.7, 124.1, 125.5, 128.1, 133.7, 135.3, 137.0, 152.1. Anal. found: C, 75.43; H, 5.96; N, 13.23. Calcd. for $\mathrm{C}_{20} \mathrm{H}_{19} \mathrm{~N}_{3} \mathrm{O}: \mathrm{C}, 75.69 ; \mathrm{H}, 6.03 ; \mathrm{N}, 13.24$.

5-(1H-Indol-3-yl)-6-phenyl-4,5-dihydro-1,2,4-triazin-3(2H)-one (4a). 2 (150 mg, $0.866 \mathrm{mmol})$ and indole (112 mg, $0.953 \mathrm{mmol})$ gave colorless crystals $4 \mathbf{a}(121 \mathrm{mg}, 48 \%)$. The reaction was carried out under varied conditions: In a mixture of methylene chloride $(5 \mathrm{~mL})$ and ethanol (2 $\mathrm{mL}$ ) with stirring for $18 \mathrm{~h}$, no product was formed; refluxing in DMF (3 mL) for $1-3 \mathrm{~h}$ formed 4a (189 $\mathrm{mg}, 75 \%)$; refluxing in acetic acid (5 mL) for $1-3 \mathrm{~h}$ provided $\mathbf{4 a}(216 \mathrm{mg}, 86 \%)$. Stirring in acetic acid $(5 \mathrm{~mL})$ in the presence of $\mathrm{Co}(\mathrm{OTf})_{2}(31 \mathrm{mg}, 0.087 \mathrm{mmol})$ at ambient temperature for $18 \mathrm{~h}$ followed by refluxing for $1 \mathrm{~h}$ furnished $4 \mathbf{a}(38 \mathrm{mg}, 15 \%) ; \mathrm{mp} 272-273{ }^{\circ} \mathrm{C} . \mathrm{R}_{\mathrm{f}}=0.45$ (EtOAc). ${ }^{1} \mathrm{H}$ NMR (400 MHz, DMSO- $\left.d_{6} \square\right): \delta 5.85$ (d, $J=2.4 \mathrm{~Hz}, 1 \mathrm{H}, 5-\mathrm{H}$, ), 6.99-7.05 (m, 2H), 7.09-7.10 (m, 1H), 7.23-7.26 (m, 3H, C6 $\left.\mathrm{H}_{5}\right), 7.30-7.33(\mathrm{~m}, 1 \mathrm{H}), 7.65-7.70$ (m, 3H), 7.73 (br.s, 1H, NH, 4-H), 10.01 (br.s, 1H, NH, 2-H), 10.87 (br.s, 1H, NH). Anal. found: C, 70.21; H, 4.80; $\mathrm{N}, 19.46$. Calcd. for $\mathrm{C}_{17} \mathrm{H}_{14} \mathrm{~N}_{4} \mathrm{O}$ : $\mathrm{C}, 70.33 ; \mathrm{H}, 4.86 ; \mathrm{N}, 19.30 .{ }^{1} \mathrm{H}$ NMR spectral data correspond to those given in the literature. ${ }^{12 \mathrm{~b}}$

5-(1-Methyl-1H-indol-3-yl)-6-phenyl-4,5-dihydro-1,2,4-triazin-3(2H)-one (4b). 2 (150 mg, $0.866 \mathrm{mmol}$ ) and 1-methylindole $(125 \mathrm{mg}, 0.953 \mathrm{mmol})$ gave colorless crystals $\mathbf{4 b}$ (166 mg, 63 $\%) ; \mathrm{mp}=265-267{ }^{\circ} \mathrm{C} . \mathrm{R}_{\mathrm{f}}=0.45$ (EtOAc). ${ }^{1} \mathrm{H}$ NMR (400 MHz, DMSO- $\left.d_{6} \square\right): \delta 3.68(\mathrm{~s}, 3 \mathrm{H}$, $\left.\mathrm{CH}_{3}\right), 5.96(\mathrm{~d}, J=3.1 \mathrm{~Hz}, 1 \mathrm{H}, 5-\mathrm{H}), 7.04-7.07$ (m, 1H, indole), 7.13-7.17 (m, 1H, indole), 7.19 (s, 1H), 7.26-7.32 (m, 3H, Ph), 7.37 (d, $J=8.2 \mathrm{~Hz}, 1 \mathrm{H}), 7.69-7.74(\mathrm{~m}, 3 \mathrm{H}), 7.84-7.85(\mathrm{~m}, 1 \mathrm{H})$, $10.27(\mathrm{~d}, J=2.0 \mathrm{~Hz}, 1 \mathrm{H}, \mathrm{NH}) .{ }^{13} \mathrm{C}$ NMR (100 MHz, DMSO-d6): $\delta 32.8,46.8,110.4,113.6$, $119.58,119.64,122.0,125.7,126.0,128.7,128.9,129.3,134.7,137.3,143.4,152.1$. Anal. found: $\mathrm{C}, 71.02 ; \mathrm{H}, 5.30 ; \mathrm{N}, 18.51$. Calcd. for $\mathrm{C}_{18} \mathrm{H}_{16} \mathrm{~N}_{4} \mathrm{O}: \mathrm{C}, 71.04 ; \mathrm{H}, 5.30 ; \mathrm{N}, 18.41$. NMR spectral data correspond to those given in the literature. ${ }^{12 \mathrm{~b}}$

5-(2-Methyl-1H-indol-3-yl)-6-phenyl-4,5-dihydro-1,2,4-triazin-3(2H)-one (4c). 2 (150 mg, $0.866 \mathrm{mmol})$ and 2-methylindole $(125 \mathrm{mg}, 0.953 \mathrm{mmol})$ gave colorless crystals $4 \mathrm{c}(127 \mathrm{mg}, 48$ $\%)$; mp 275-276 ${ }^{\circ} \mathrm{C} . \mathrm{R}_{\mathrm{f}}=0.4$ (EtOAc). ${ }^{1} \mathrm{H}$ NMR (400 MHz, DMSO-d6 $\left.\square\right): \delta 2.46\left(\mathrm{~s}, 3 \mathrm{H}, \mathrm{CH}_{3}\right)$, $5.99(\mathrm{~d}, J=2.3 \mathrm{~Hz}, 1 \mathrm{H}, 5-\mathrm{H}), 6.91-6.99(\mathrm{~m}, 2 \mathrm{H}$, indole), 7.20-7.28 (m, 4H), 7.47 (d, $J=7.8 \mathrm{~Hz}$, 1H), 7.59-7.62 (m, 3H), 10.32 (d, $J=1.7 \mathrm{~Hz}, 1 \mathrm{H}, \mathrm{NH}), 10.99$ (s, 1H, NH). ${ }^{13} \mathrm{C} \mathrm{NMR}(100 \mathrm{MHz}$, DMSO-d6): $\delta 11.8,47.1,111.1,111.4,118.3,119.4,120.9,125.9,126.3,128.7,129.1,134.0$, 135.3, 135.6, 142.4, 151.2. Anal. found: $\mathrm{C}, 71.30 ; \mathrm{H}, 5.30 ; \mathrm{N}, 18.48$. Calcd. for $\mathrm{C}_{18} \mathrm{H}_{16} \mathrm{~N}_{4} \mathrm{O}$ : C, 71.04; H, 5.30; N, 18.41. NMR spectral data correspond to those given in the literature. ${ }^{12 \mathrm{~b}}$

\section{General method for the preparation of $(4 \mathrm{~d}-\mathrm{g})$}

A mixture of 6-phenyl-1,2,4-triazin-3(2H)-one $(2,200 \mathrm{mg}, 1.15 \mathrm{mmol})$, the nucleophilic reactant (1.1 mmol equiv.), and $\mathrm{BF}_{3} \bullet \mathrm{OMe}_{2}(145 \mu \mathrm{L}, 1.15 \mathrm{mmol})$ in acetic acid $(5 \mathrm{~mL})$ was refluxed for 1-3 h. The reaction was monitored by TLC. The reaction mixture was evaporated in vacuo; the gummy residue was crystallized from ethanol. 
6-Phenyl-5-(thiophen-2-yl)-4,5-dihydro-1,2,4-triazin-3(2H)-one (4d). 2 and thiophene (107 $\mathrm{mg}, 1.27 \mathrm{mmol})$ gave cream colored crystals $4 \mathbf{d}(122 \mathrm{mg}, 41 \%)$; mp 250-251 ${ }^{\circ} \mathrm{C} .{ }^{1} \mathrm{H}$ NMR (400 MHz, DMSO- $\left.d_{6} \square\right): \delta 6.03(\mathrm{~d}, J=3.1 \mathrm{~Hz}, 1 \mathrm{H}, 5-\mathrm{H}), 6.87-6.97(\mathrm{~m}, 1 \mathrm{H}$, thiophene), 7.01 (d, $J=$ $2.8 \mathrm{~Hz}, 1 \mathrm{H}$, thiophene), 7.36-7.42 (m, 4H, Ph, thiophene), 7.76-7.78 (m, 2H, Ph), $8.16(\mathrm{~s}, 1 \mathrm{H}$, $\mathrm{NH}), 10.34(\mathrm{~s}, 1 \mathrm{H}, \mathrm{NH}) .{ }^{13} \mathrm{C}$ NMR (100 MHz, DMSO-d $): \delta 49.0,126.2,126.3,126.8,127.4$, 129.1, 129.7, 134.0, 143.3, 143.7, 152.0. Anal. found: C, 60.74; H, 4.40; N, 16.45. Calcd. for $\mathrm{C}_{13} \mathrm{H}_{11} \mathrm{~N}_{3} \mathrm{OS}: \mathrm{C}, 60.68 ; \mathrm{H}, 4.31 ; \mathrm{N}, 16.33$.

5-(2-Oxo-2-phenylethyl)-6-phenyl-4,5-dihydro-1,2,4-triazin-3(2H)-one $\quad(4 e), \quad 2 \quad$ and acetophenone (153 mg, $1.27 \mathrm{mmol}$ ) gave cream colored crystals $4 \mathbf{e}(200 \mathrm{mg}, 59 \%)$; mp $204{ }^{\circ} \mathrm{C}$. $\mathrm{R}_{\mathrm{f}}=0.7$ (EtOAc). ${ }^{1} \mathrm{H}$ NMR (400 MHz, DMSO-d6) $\square: \delta 2.99(\mathrm{dd}, J=17.2,2.9 \mathrm{~Hz}, 1 \mathrm{H}), 3.55$ (dd, $J=17.2,9.2 \mathrm{~Hz}, 1 \mathrm{H}), 5.17(\mathrm{dt}, J=3.6,5.1 \mathrm{~Hz}, 1 \mathrm{H}, 5-\mathrm{H}), 7.25-7.30(\mathrm{~m}, 1 \mathrm{H}), 7.32-7.41(\mathrm{~m}, 3 \mathrm{H})$, 7.45-7.49 (m, 2H), 7.56-7.62 (m, 1H), 7.66-7.75 (m, 2H), 7.91-7.94 (m, 2H), 10.04 (d, J = 2.2 $\mathrm{Hz}, 1 \mathrm{H}, \mathrm{NH}) .{ }^{13} \mathrm{C}$ NMR $\left(100 \mathrm{MHz}, \mathrm{DMSO}-d_{6}\right): \delta 40.9,46.2,125.4,128.2,128.7,129.3,133.3$, 133.5, 136.5, 143.6, 151.9, 196.8. Anal. found: C, 69.75; H, 5.12; N, 14.43. Calcd. for $\mathrm{C}_{17} \mathrm{H}_{15} \mathrm{~N}_{3} \mathrm{O}_{2}$ : C, 69.61; H, 5.15; N, 14.33.

5-[2-Oxo-2-(thiophen-2-yl)ethyl]-6-phenyl-4,5-dihydro-1,2,4-triazin-3(2H)-one (4f). 2 and 2acetothienone (160 mg, $1.27 \mathrm{mmol}$ ) gave cream colored crystals $4 \mathbf{f}$ (142 mg, 41\%); mp 205-207 ${ }^{\circ} \mathrm{C} .{ }^{1} \mathrm{H}$ NMR (400 MHz, DMSO- $\left.d_{6} \square\right)$ : $\delta$ 2.95-3.03 (m, 1H), 3.36-3.46 (m, 1H), 5.15 (m, 1H, 5$\mathrm{H}), 7.18-7.22(\mathrm{~m}, 1 \mathrm{H}), 7.38-7.45(\mathrm{~m}, 3 \mathrm{H}), 7.57$ (br.s, 1H), 7.68-7.72 (m, 2H), 7.87-7.90 (m, 1H), 7.97-7.99 (m, 1H), 10.11 (br.s, 1H). Anal. found: C, 60.32; H, 4.44; N, 14.17. Calcd. for $\mathrm{C}_{15} \mathrm{H}_{13} \mathrm{~N}_{3} \mathrm{O}_{2} \mathrm{~S}: \mathrm{C}, 60.18 ; \mathrm{H}, 4.38 ; \mathrm{N}, 14.04$.

5-(2-Oxopropyl)-6-phenyl-4,5-dihydro-1,2,4-triazin-3(2H)-one (4g). 2 and acetone (74 $\mathrm{mg}$, $1.27 \mathrm{mmol})$ gave colorless crystals $4 \mathrm{~g}(112 \mathrm{mg}, 42 \%)$; mp $141{ }^{\circ} \mathrm{C} . \mathrm{R}_{\mathrm{f}}=0.45$ (EtOAc). ${ }^{1} \mathrm{H}$ NMR (400 MHz, DMSO-d6): $\delta 2.15\left(\mathrm{~s}, 3 \mathrm{H}, \mathrm{CH}_{3}\right), 2.50-2.53(\mathrm{~m}, 1 \mathrm{H}), 2.87(\mathrm{dd}, J=16.6,9.6 \mathrm{~Hz}, 1 \mathrm{H})$, 4.90-5.04 (m, 1H, 5-H), 7.21-7.29 (m, 1H, NH), 7.30-7.43 (m, 3H, Ph), 7.66-7.69 (m, 2H, Ph), 9.99 (d, $J=2.1 \mathrm{~Hz}, 1 \mathrm{H}, \mathrm{NH}$ ). Anal. found: $\mathrm{C}, 62.38 ; \mathrm{H}, 5.76 ; \mathrm{N}, 18.29$. Calcd. for $\mathrm{C}_{12} \mathrm{H}_{13} \mathrm{~N}_{3} \mathrm{O}_{2}$ : C, $62.33 ; \mathrm{H}, 5.67 ; \mathrm{N}, 18.17$. NMR spectral data correspond to those given in the literature. ${ }^{12 \mathrm{a}}$

General procedure for the synthesis of 2-alkyl-5-(2-oxopropyl)-6-phenyl-4,5-dihydro-1,2,4triazin-3(2H)-ones (5a-d)

Acetone $(5 \mathrm{~mL})$ was added to a suspension of 6-phenyl-1,2,4-triazin-3(2H)-one $(2,1.00 \mathrm{~g}, 5.77$ mmol) in aqueous $\mathrm{NaOH}(2 \mathrm{~N}, 5 \mathrm{~mL})$. To the resulting solution was added the appropriate alkyl halide (see Table 3). After stirring for $24 \mathrm{~h}$, the precipitate formed was filtered off and crystallized from acetonitrile.

2-Methyl-5-(2-oxopropyl)-6-phenyl-4,5-dihydro-1,2,4-triazin-3(2H)-one (5a). 2 and methyl iodide gave colorless crystals 5a (482 mg, 34\%); mp 136-137 ${ }^{\circ} \mathrm{C}$. ${ }^{1} \mathrm{H}$ NMR (400 MHz, DMSO$\left.d_{6}\right): \delta 2.14\left(\mathrm{~s}, 3 \mathrm{H}, \mathrm{CH}_{3} \mathrm{CO}\right), 2.49-2.54(\mathrm{~m}, 1 \mathrm{H}, \mathrm{CH}), 2.82-2.89(\mathrm{~m}, 1 \mathrm{H}, \mathrm{CH}), 3.29\left(\mathrm{~s}, 3 \mathrm{H}, \mathrm{NCH}_{3}\right)$, 4.96-5.00 (m, 1H, CH, 5-H), 7.35-7.40 (m, 4H, Ph), 7.68-7.70 (m, 2H, Ph). ${ }^{13} \mathrm{C}$ NMR (100 MHz, DMSO- $\left.d_{6}\right): \delta 30.3,37.0,45.3,46.1,125.4,128.7,129.4,132.6,143.9,151.7,205.4$. Anal. found: C 63.62; $\mathrm{H}$ 5.98; $\mathrm{N}$ 17.05. Calcd. for $\mathrm{C}_{13} \mathrm{H}_{15} \mathrm{~N}_{3} \mathrm{O}_{2}$ : C 63.66; $\mathrm{H} 6.16 ; \mathrm{N} 17.13$. 
2-Ethyl-5-(2-oxopropyl)-6-phenyl-4,5-dihydro-1,2,4-triazin-3(2H)-one (5b). 2 and ethyl iodide gave colorless crystals $5 \mathbf{b}(569 \mathrm{mg}, 38 \%)$; mp 146-147 ${ }^{\circ} \mathrm{C} .{ }^{1} \mathrm{H}$ NMR $(400 \mathrm{MHz}$, DMSO$d_{6} \square: \delta 1.17\left(\mathrm{t}, J=6.9 \mathrm{~Hz}, 3 \mathrm{H}, \mathrm{CH}_{3}\right), 2.12\left(\mathrm{~s}, 3 \mathrm{H}, \mathrm{CH}_{3} \mathrm{CO}\right), 2.56(\mathrm{dd}, J=16.3,2.1 \mathrm{~Hz}, 1 \mathrm{H}, \mathrm{CH})$, 2.80 (dd, $J=16.4,9.2 \mathrm{~Hz}, 1 \mathrm{H}, \mathrm{CH}), 3.64-3.72\left(\mathrm{~m}, 2 \mathrm{H}, \mathrm{NCH}_{2}\right), 4.98-5.00$ (m, 1H, 5-H), 7.42$7.44(\mathrm{~m}, 3 \mathrm{H}, \mathrm{Ph}), 7.51(\mathrm{~d}, J=3.2 \mathrm{~Hz}, 1 \mathrm{H}, \mathrm{NH}), 7.71-7.73(\mathrm{~m}, 2 \mathrm{H}, \mathrm{Ph}) .{ }^{13} \mathrm{C} \mathrm{NMR}(100 \mathrm{MHz}$, DMSO-d6): $\delta 13.3,30.3,43.3,45.4,46.0,125.4,128.7,129.4,132.8,143.9,151.0,205.4$. Anal. found: C 64.88; H 6.77; $\mathrm{N}$ 16.07. Calcd. for $\mathrm{C}_{14} \mathrm{H}_{17} \mathrm{~N}_{3} \mathrm{O}_{2}$ : C 64.85; H 6.61; $\mathrm{N} 16.20$.

5-(2-Oxopropyl)-6-phenyl-2-propyl-4,5-dihydro-1,2,4-triazin-3(2H)-one (5c). 2 and propyl bromide gave colorless crystals 5c (190 mg, 12\%); mp 129-130 ${ }^{\circ} \mathrm{C}$. ${ }^{1} \mathrm{H}$ NMR (400 MHz, DMSO- $d_{6} \square: \delta 0.89\left(\mathrm{t}, J=7.4 \mathrm{~Hz}, 3 \mathrm{H}, \mathrm{CH}_{3}\right), 1.60-1.69\left(\mathrm{~m}, 2 \mathrm{H}, \mathrm{CH}_{2}\right), 2.12\left(\mathrm{~s}, 3 \mathrm{H}, \mathrm{COCH}_{3}\right)$, $2.57(\mathrm{dd}, J=16.4,3.1 \mathrm{~Hz}, 1 \mathrm{H}, \mathrm{CH}), 2.78(\mathrm{dd}, J=16.4,9.1 \mathrm{~Hz}, 1 \mathrm{H}, \mathrm{CH}), 3.51-3.71(\mathrm{~m}, 2 \mathrm{H}$, $\left.\mathrm{CH}_{2}\right), 4.99(\mathrm{td}, J=9.1,3.5,3.5 \mathrm{~Hz}, 1 \mathrm{H}, 5-\mathrm{H}), 7.36-7.46(\mathrm{~m}, 3 \mathrm{H}, \mathrm{Ph}), 7.49(\mathrm{~d}, J=3.8 \mathrm{~Hz}, 1 \mathrm{H}$, $\mathrm{NH}), 7.70-7.72(\mathrm{~m}, 2 \mathrm{H}, \mathrm{Ph}) .{ }^{13} \mathrm{C}$ NMR (100 MHz, DMSO-d $)$ : $\delta$ 11.0, 21.2, 30.3, 45.6, 46.0, 49.8, 125.4, 128.7, 129.3, 132.8, 143.6, 151.0, 205.4. Anal. found: C 65.82; H 7.13; N 15.49. Calcd. for $\mathrm{C}_{15} \mathrm{H}_{19} \mathrm{~N}_{3} \mathrm{O}_{2}$ : C 65.91; $\mathrm{H} 7.01 ; \mathrm{N} 15.37$.

2-Benzyl-5-(2-oxopropyl)-6-phenyl-4,5-dihydro-1,2,4-triazin-3(2H)-one (5d). 2 and benzyl chloride gave colorless crystals $5 \mathbf{d}(260 \mathrm{mg}, 14 \%)$; mp $162{ }^{\circ} \mathrm{C}$. ${ }^{1} \mathrm{H}$ NMR (400 MHz, DMSO- $\left.d_{6}\right)$ : $\delta 2.14\left(\mathrm{~s}, 3 \mathrm{H}, \mathrm{COCH}_{3}\right), 2.56(\mathrm{dd}, J=16.6,2.9 \mathrm{~Hz}, 1 \mathrm{H}, \mathrm{CH}), 2.84(\mathrm{dd}, J=16.6,9.4 \mathrm{~Hz}, 1 \mathrm{H}, \mathrm{CH})$, 4.80-4.92 (m, 2H, CH $), 5.02(\mathrm{td}, J=9.3,3.1,3.1 \mathrm{~Hz}, 1 \mathrm{H}, 5-\mathrm{H}), 7.17-7.25(\mathrm{~m}, 1 \mathrm{H}, \mathrm{Ph}), 7.26-$ $7.40(\mathrm{~m}, 7 \mathrm{H}, \mathrm{Ph}), 7.44(\mathrm{~d}, J=3.7 \mathrm{~Hz}, 1 \mathrm{H}, \mathrm{NH}), 7.65-7.67(\mathrm{~m}, 2 \mathrm{H}, \mathrm{Ph}) .{ }^{13} \mathrm{C} \mathrm{NMR}(100 \mathrm{MHz}$, DMSO-d $)_{6}: \delta 30.3,45.8,46.2,51.8,125.4,126.8,127.6,128.2,128.7,129.5,132.6,138.6$, 144.1, 151.1, 205.3. Anal. found: C 71.32; $\mathrm{H}$ 5.84; $\mathrm{N} 13.11$. Calcd. for $\mathrm{C}_{19} \mathrm{H}_{19} \mathrm{~N}_{3} \mathrm{O}_{2}$ : C 71.01; $\mathrm{H}$ 5.96; N 13.08. ${ }^{1} \mathrm{H}$ NMR spectral data correspond to those given in the literature. ${ }^{12 \mathrm{a}}$

\section{Acknowledgements}

We are pleased to thank Dr. P. Slepukhin for assistance with the X-ray analysis. This work was supported by the program of the Russian Federation President for government support of young scientists, MK-1157.2009.3, and by the program on carrying out of scientific researches by candidates of science and post-graduate students of Ural Federal University.

\section{References and Notes}

1. Rewcastle G. W. In Comprehensive Heterocyclic Chemistry III; Elsevier:Oxford, 2008; Vol. 8, pp 117-272.

2. Gacek, M.; Undheim, K.; Oftebro, R.; Laland, S. G. FEBS Letters 1979, 98, 355.

3. Barchi, J. J.; Musser, S.; Marquez, V. E. J. Org. Chem. 1992, 57, 536.

4. (a) Schroeder, A. C.; Bardos, T. J. J. Med. Chem. 1981, 24, 109. (b) Efange, S. M. N.; Alessi, 
E. M.; Shih, H. C.; Cheng, Y.-C.; Bardos, T. J.; J. Med. Chem. 1985, 28, 904. (c) Efange, S. M. N.; Cheng, Y.-C.; Bardos, T. J. Nucleosides \& Nucleotides 1985, 4, 545.

5. (a) Wotring, L. L.; Townsend, L. B.; Cancer Res. 1989, 49, 289. (b) Raić-Malić, S.; Grdiša, M.; Pavelic, K.; Mintas, M. Eur. J. Med. Chem. 1999, 34, 405. (c) Mischra, R.C.; Dwivedi, N., Tripathi, R. P.; Bansal, I.; Saxena, J. K. Nucleosides, Nucleotides \& Nucleic Acids 2005, $24,15$.

6. (a) Morrey, J. D.; Smee, D. F.; Sidwell, R. W.; Tseng, C. Antiviral Res. 2002, 55, 107. (b) Sharma, A. P.; Ollapally, A. P.; Jones, W.; Lemon, T. Nucleosides, Nucleotides \& Nucleic Acids 1992, 11, 1009. (c) Alexeeva, I.; Dyachenko, N.; Nosach, L.; Zhovnovataya, V.; Rybalko, S.; Lozitskaya, R.; Fedchuk, A.; Lozitsky, V.; Gridina, T.; Shalamay, A.; Palchikovskaja, L.; Povnitsa, O. Nucleosides, Nucleotides \& Nucleic Acids 2001, 20, 1147. (d) Kabbaj, Y.; Lazrek, H. B.; Barascut, J. L.; Imbach, J. L. Nucleosides, Nucleotides \& Nucleic Acids 2005, 24, 161. (e) Holy, A.; Guenter, J.; Dvorakova, H.; Masojidkova, M.; Andrei, G.; Snoeck, R.; Balzarini, J.; De Clercq, E. J. Med. Chem. 1999, 42, 2064. (f) Maslen, H.L.; Hughes, D.; Hursthouse, M.; De Clercq, E.; Balzarini, J.; Simons, C. J. Med. Chem. 2004, 47, 5482.

7. (a) Khalil, N. S. A. M.; Mansour, A. K.; Eid, M.M. Nucleosides, Nucleotides \& Nucleic Acids 2004, 23, 1889. (b) Modzelewska-Banachiewicz, B.; Kaminska, T. Eur. J. Med. Chem. 2001, 36, 93 .

8. (a) Chupakhin, O.N.; Charushin, V.N.; van der Plas, H.C. In Nucleophilic Aromatic Substitution of Hydrogen, Academic Press: San Diego, 1994, p 367; (b) Charushin, V.N.; Chupakhin, O.N. Mendeleev Commun. 2007, 17, 249.

9. (a) Gundersen, L.-L.; Rise, F.; Undheim, K. Tetrahedron 1992, 48, 5647. (b) Rise, F.; Romming, C.; Undheim, K. Acta Chem. Scand. B 1985, 459. (c) Rise, F.; Undheim, K. Acta Chem. Scand. B 1985, 39, 195. (d) Coppola, G. M.; Fraser, J. D.; Hardtmann, G. E.; Huegi, B. S. J. Heterocycl. Chem. 1979, 545. (e) Kashima, C.; Katoh, A.; Yokota, Y.; Omote, Y. J. Chem. Soc., Perkin Trans. I 1981, 489. (f) Singh, K.; Arora, D.; Falkowski, D.; Liu, Q.; Moreland, R. S. Eur. J. Org. Chem. 2009, 3258. (g) Singh, K.; Arora, D.; Singh, S. Tetrahedron Lett. 2007, 48, 1349.

10. Shanmugam, P.; Boobalan, P.; Perumal, P.T. Tetrahedron 2007, 63, 12215.

11. (a) Zyryanov, G. V.; Pilitcheva, T. L.; Egorov, I. N.; Rusinov, V. L.; Chupakhin, O. N.; Russ. J. Org. Chem. (Engl. Transl.) 2000, 36, 602. (b) Rusinov, V. L.; Zyryanov, G. V.; Egorov I. N.; Ulomsky, E. N.; Alexandrov, G. G.; Chupakhin, O. N. Russ. J. Org. Chem. (Engl. Transl.) 2004, 40, 85.

12. (a) Rasmussen, A.; Rise, F.; Undheim K. Acta Chem. Scand. 1985, 39B, 235. (b) Chupakhin, O. N.; Egorov, I. N.; Rusinov, V. L.; Slepukhin, P. A. Izv. Acad. Nauk, Ser. Khim. 2010, 970; [Russ. Chem. Bull. Int. Ed. 2010, 59, 991].

13. Girke, W. P. K. Chem. Ber. 1979, 112, 1. 
14. (a) Esquivias, J.; Arrayás, R. G.; Carretero, J. C. Angew. Chem. Int. Ed. 2006, 45, 629. (b) Poulsen, T. B.; Jørgensen, K. A. Chem. Rev. 2008, 108, 2903. (c) Li, B.-Y.; Li, Z.-J.; Meng, X.-B. Carbohydrate Res. 2010, 345, 1708.

15. (a) Shirakawa, S.; Kobayashi, S. Org. Lett. 2006, 8, 4939. (b) Terada, M.; Yokoyama, S.; Sorimachi, K.; Uraguchi, D. Adv. Synth. Catal. 2007, 349, 1863; (c) Terada, M. Synthesis 2010, 1929.

16. Rasmussen, A.; Rise, F.; Undheim, K. Acta Chem. Scand. 1985, 39B, 235.

17. Crystallographic data have been deposited with the Cambridge Crystallographic Data Centre and can be obtained via: www.ccdc.cam.ac.uk/data_request/cif. Deposition numbers: (a) CCDC 806022 for 4e. (b) CCDC 752696 for $\mathbf{5 d}$.

18. Coppola, G. M.; Hardtmann, G. E.; Huegi, B. S. J. Heterocycl. Chem. 1974, 51

19. Lalezari, I.; Sharghi, N.; Shafiee, A.; Yalpani, M. J. Heterocycl. Chem. 1969, 403.

20. CRYSALYSPRO, CRYSALYSRED (version 1.171.31.8). Oxford Diffraction Ltd., Abingdon, Oxfordshire (England) 2007.

21. Sheldrick, G. M. SHELXS/L-97, Programs for Crystal Structure Determination, University of Göttingen, Göttingen (Germany) 1997. See also: Sheldrick, G. M. Acta Crystallogr. 1990, A46, 467; Acta Crystallogr. 2008, A64, 112.

22. Rupe, K. Helv. Chim. Acta 1927, 10, 305.

23. Krecmerova, M.; Hrebabecky, H.; Masojidkova, M.; Holy, A. Coll. Czech. Chem. Commun. 1996, 61, 458. 